\title{
Magnetite: a search for the half-metallic state
}

\author{
M Fonin ${ }^{1}$, Yu S Dedkov ${ }^{2}, \mathbf{R}$ Pentcheva $^{3}$, U Rüdiger $^{1}$ and G Güntherodt ${ }^{4}$ \\ ${ }^{1}$ Fachbereich Physik, Universität Konstanz, 78457 Konstanz, Germany \\ ${ }^{2}$ Institut für Festkörperphysik, Technische Universität Dresden, 01062 Dresden, Germany \\ ${ }^{3}$ Department of Earth and Environmental Sciences, University of Munich, Theresienstraße 41, \\ 80333 Munich, Germany \\ ${ }^{4}$ II. Physikalisches Institut, Rheinisch-Westfälische Technische Hochschule Aachen, \\ 52056 Aachen, Germany \\ E-mail: mikhail.fonin@uni-konstanz.de
}

\begin{abstract}
We present a detailed study of the spin-dependent electronic structure of thin epitaxial magnetite films of different crystallographic orientations. Using spin- and angle-resolved photoelectron spectroscopy at room temperature, we determine for epitaxial $\mathrm{Fe}_{3} \mathrm{O}_{4}(111)$ films a maximum spin polarization value of $-(80 \pm 5) \%$ near $E_{\mathrm{F}}$. The spin-resolved photoelectron spectra for binding energies between $1.5 \mathrm{eV}$ and $E_{\mathrm{F}}$ show good agreement with the spin-split band structure from density functional theory (DFT) calculations which predict an overall energy gap in the spin-up electron bands in high symmetry directions, thus providing evidence for the half-metallic ferromagnetic state of $\mathrm{Fe}_{3} \mathrm{O}_{4}$ in the [111] direction. In the case of the $\mathrm{Fe}_{3} \mathrm{O}_{4}(100)$ surface, both the spin-resolved photoelectron spectroscopy experiments and the DFT density of states give evidence for a half-metal to metal transition: the measured spin polarization of about $-(55 \pm 10) \%$ at $E_{\mathrm{F}}$ and the theoretical value of $-40 \%$ are significantly lower than the $-100 \%$ predicted by local spin density approximation (LSDA) calculations for the bulk magnetite crystal as well as the $-(80 \pm 5) \%$ obtained for the $\mathrm{Fe}_{3} \mathrm{O}_{4}$ (111) films. The experimental findings were corroborated by DFT calculations as due to a surface reconstruction leading to the electronic states in the majority-spin band gap and thus to the reduced spin polarization.
\end{abstract}

(Some figures in this article are in colour only in the electronic version)

\section{Introduction}

Discovered about $1500 \mathrm{BC}$, magnetite $\left(\mathrm{Fe}_{3} \mathrm{O}_{4}\right)$ was the first known magnetic material. Since then a large number of magnetic materials have been discovered, nevertheless magnetite remains one of the most intensively studied magnetic compounds. Recently, the interest in $\mathrm{Fe}_{3} \mathrm{O}_{4}$ was re-inspired by the prediction of the half-metallic ferromagnetic state $[1,2]$ resulting 

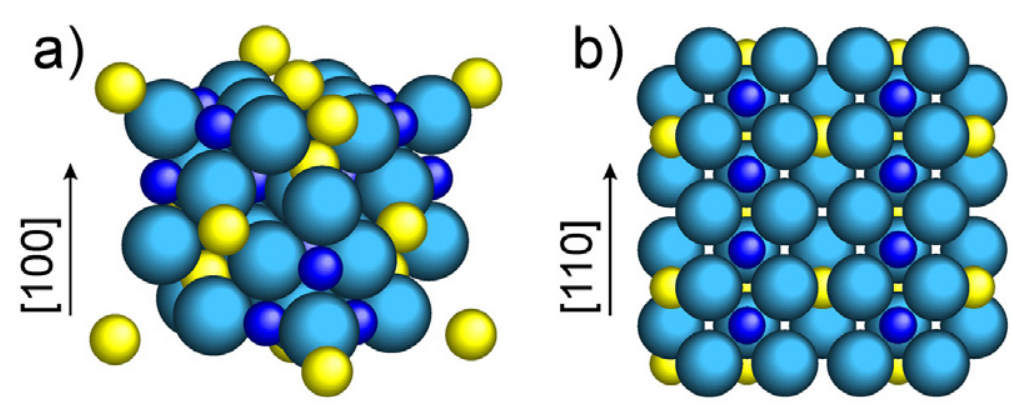

FeA-site $\odot$ Fe B-site

Figure 1. (a) Perspective side view of the $\mathrm{Fe}_{3} \mathrm{O}_{4}$ crystal structure. In (b) top view of the $\mathrm{Fe}_{3} \mathrm{O}_{4}(100)$ bulk B-termination is shown.

in a $100 \%$ spin polarization at the Fermi energy which makes magnetite a promising material for magneto- and spinelectronics.

Intensive investigation of the transport properties of magnetite began after the fundamental discovery made by Verwey, who observed a sharp, first-order phase transition (Verwey transition) upon cooling below $120 \mathrm{~K}$ at which the resistivity of magnetite decreases by two orders of magnitude [3-5]. Verwey and co-workers [4, 5] proposed a model in which this phase transition is associated with an electron localization-delocalization transition on the $\mathrm{B}$ sites. The $\mathrm{Fe}^{2+}$ ion at the $\mathrm{B}$ site can be described as an 'extra' electron plus an $\mathrm{Fe}^{3+}$ ion. The relatively high electrical conductivity of magnetite at room temperature is due to the hopping of the 'extra' electron between equivalent $\mathrm{B}$ sites. Cooling below $T_{\mathrm{V}}$ freezes these 'extra' electrons and causes their ordering at the $\mathrm{Fe}^{2+} \mathrm{B}$ sites leading to the configuration where the (001) planes are alternately occupied by $\mathrm{Fe}_{\mathrm{B}}^{2+}$ and $\mathrm{Fe}_{\mathrm{B}}^{3+}$ cations. The orthorhombic superstructure model proposed by Verwey was apparently confirmed by neutron diffraction [6], but further experiments showed the symmetry of the low temperature unit cell to be monoclinic [7] and disproved the Verwey model. The observation of monoclinic symmetry was also concluded from a single crystal x-ray study [8]. However, in the more recent studies some small deviations from the monoclinic cell have been reported $[9,10]$. As the issue of definitive, experimentally confirmed crystallographic structure of the low-temperature phase remains controversial, many theoretical models for charge ordering have been proposed: purely electronic [11-13], electron-phonon coupled [14-16], as well as a bond dimerized ground state without charge separation [17].

Above the Verwey transition, magnetite crystallizes in the cubic inverse spinel structure with space group $F d \overline{3} m(a=8.396 \AA)$. The iron cations $\left(\mathrm{Fe}^{2+}\right.$ and $\left.\mathrm{Fe}^{3+}\right)$ are located in the interstitial sites of a close-packed face-centred cubic (fcc) sublattice which is formed by the oxygen anions $\left(\mathrm{O}^{2-}\right)$ [18]. Two different kinds of cation sites exist in the crystal: the first (A) is tetrahedrally coordinated by oxygen and occupied only by $\mathrm{Fe}^{3+}$ ions, and the second (B) is octahedrally coordinated by oxygen and occupied by an equal number of randomly distributed $\mathrm{Fe}^{2+}$ and $\mathrm{Fe}^{3+}$ ions, resulting in an average valence value of $2.5+$ per $\mathrm{Fe}_{\mathrm{B}}$ cation (figure 1).

Magnetite is a ferrimagnet with an extraordinary high transition temperature $T_{\mathrm{C}}=858 \mathrm{~K}$. The A- and B-site magnetic moments are antiferromagnetically coupled and the d-orbital occupation can be represented as $\left(\mathrm{t}_{2 \mathrm{~g}}^{3} \mathrm{e}_{\mathrm{g}}^{2}\right) \downarrow$ at A sites and $\left(\mathrm{t}_{2 \mathrm{~g}}^{3} \mathrm{e}_{\mathrm{g}}^{2}\right) \uparrow\left(\mathrm{t}_{2 \mathrm{~g}}^{0.5}\right) \downarrow$ at B sites, leading to the half-metallic state with integer magnetic moment of $4 \mu_{\mathrm{B}}$ per $\mathrm{Fe}_{3} \mathrm{O}_{4}$ formula unit. 
The electronic structure of $\mathrm{Fe}_{3} \mathrm{O}_{4}$ was studied theoretically by several groups [1, 2, 19-22]. Zhang and Satpathy [2] performed band structure calculations using the local spin density approximation (LSDA) to the density-functional theory (DFT) and the 'constrained' densityfunctional methods. This theoretical study showed that the $\mathrm{O} 2 \mathrm{p}$ orbitals lie well below $E_{\mathrm{F}}$, with the electron bands near $E_{\mathrm{F}}$ consisting primarily of Fe $3 \mathrm{~d}$ orbitals. This result is in agreement with the earlier augmented plane wave (APW) calculations [1]. The most interesting feature of the calculated spin-polarized band structure is the fact that the majority spin electrons ( $\uparrow$ ) exhibit insulating or semiconducting behaviour while the minority spin electrons $(\downarrow)$ show metallic behaviour [2]. Thus, only spin-down electrons were found to be present at $E_{\mathrm{F}}$, leading to the $-100 \%$ spin polarization and moreover, the d electrons at $E_{\mathrm{F}}$ have predominantly B site character. The spin-down character of the electrons at $E_{\mathrm{F}}$ was found to be in good agreement with earlier APW calculations [1] as well as with early spin-polarized photoemission experiments [23].

The experimental studies concerning the spin-resolved electronic structure of $\mathrm{Fe}_{3} \mathrm{O}_{4}$ were performed mostly by means of photoelectron spectroscopy (PES) [23-31]. Due to a very low conduction of $\mathrm{Fe}_{3} \mathrm{O}_{4}$ below $T_{\mathrm{V}}=120 \mathrm{~K}$ the spin-resolved electronic structure of $\mathrm{Fe}_{3} \mathrm{O}_{4}$ cannot be studied by any superconductor spin-probing method, making spin-resolved PES [23-31] one of the most appropriate measurement techniques.

Alvarado et al [23-25] performed the first spin-resolved PES measurements on $\mathrm{Fe}_{3} \mathrm{O}_{4}(100)$ single crystals. The spin polarization $P(h v)$ of the photoelectrons with energies up to $11.2 \mathrm{eV}$ was analysed by Mott scattering. $P(h v)=\left(n_{\uparrow}-n_{\downarrow}\right) /\left(n_{\uparrow}+n_{\downarrow}\right)$, where $n_{\uparrow}\left(n_{\downarrow}\right)$ is the number of spin-up (spin-down) photoelectrons obtained at the photon energy $h v$. The maximum spin polarization value of $-60 \%$ near $E_{\mathrm{F}}$ was measured at around $5 \mathrm{eV}$ photon energy at $10 \mathrm{~K}$ [24]. The experimentally obtained spin polarization values were compared with those predicted by the single-ion-in-a-crystal-field (SICF) model. As derived in this model, the maximum obtainable spin polarization at $T=0 \mathrm{~K}$ is $P=-66.6 \%[24,25]$ which is in agreement with the measured value of $-60 \%$. However, this spin polarization value is obviously much lower than $-100 \%$ predicted on the basis of LSDA calculations [1, 2]. Recently, spin-resolved photoemission spectroscopy measurements of epitaxial $\mathrm{Fe}_{3} \mathrm{O}_{4}(111)$ thin films grown on the $\mathrm{Fe}(110)$ surface were performed by Kim et al [26]. A larger photoemission intensity at $E_{\mathrm{F}}$ for the $\mathrm{Fe}_{3} \mathrm{O}_{4}(111) / \mathrm{Fe}$ (110) films was found comparing to the bulk $\mathrm{Fe}_{3} \mathrm{O}_{4}$ samples. This higher photoemission intensity was attributed to the possible formation of an oxygen deficient $\mathrm{Fe}_{4} \mathrm{O}_{4}$ surface with respect to the bulk-terminated one. The observation of relatively small spin polarization values about $+16 \%$ at $E_{\mathrm{F}}$ supports the fact that the films do not have the pure $\mathrm{Fe}_{3} \mathrm{O}_{4}$ stoichiometry. As for the (100)-oriented $\mathrm{Fe}_{3} \mathrm{O}_{4}$ surface, the spin-resolved photoemission measurements of thin epitaxial films grown on $\mathrm{MgO}(100)$ substrates performed at room temperature yielded spin polarization values of about - (40-55)\% near $E_{\mathrm{F}}[27,28]$ which are still significantly lower than theoretically predicted by LSDA calculations. This large discrepancy was claimed to result either from sample surface imperfections [27] or from strong electron correlation effects [28].

In this article we present the experimental results of spin-polarized electronic structure investigations of epitaxial $\mathrm{Fe}_{3} \mathrm{O}_{4}(111)$ and $\mathrm{Fe}_{3} \mathrm{O}_{4}(100)$ thin films. The latter are presented in the light of recent DFT calculations for the $\mathrm{Fe}_{3} \mathrm{O}_{4}(100)$ surface. The problems of defining and measuring spin polarization of half-metallic ferromagnets are discussed in the context of $\mathrm{Fe}_{3} \mathrm{O}_{4}$.

\section{Spin-resolved photoelectron spectroscopy of $\mathrm{Fe}_{3} \mathrm{O}_{4}(111)$}

High-quality epitaxial $\mathrm{Fe}_{3} \mathrm{O}_{4}(111)$ thin films were prepared on the W(110) as well as on the $\mathrm{Mo}(110) / \mathrm{Al}_{2} \mathrm{O}_{3}(11 \overline{2} 0)$ substrates by in situ oxidation of thin epitaxial $\mathrm{Fe}(110)$ films $[29,30]$. 

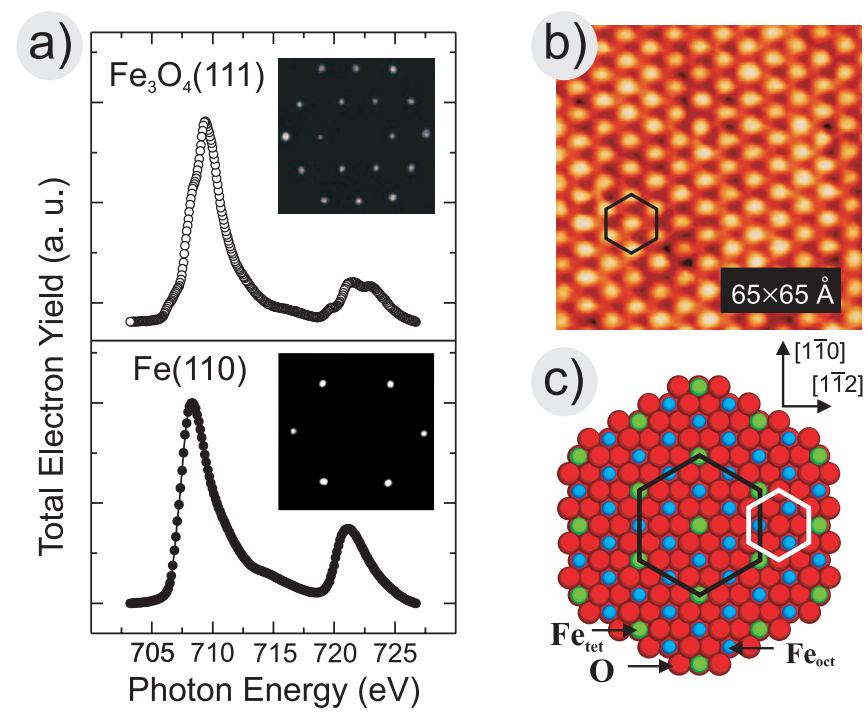

Figure 2. In (a) the $\mathrm{Fe} \mathrm{L}_{2,3}$-edge XAS spectra of an epitaxial $\mathrm{Fe}_{3} \mathrm{O}_{4}(111)$ film and an epitaxial $\mathrm{Fe}(110)$ film are presented together with the corresponding LEED patterns. In (b) an atomically resolved STM image of the $\mathrm{Fe}_{3} \mathrm{O}_{4}(111)$ surface is shown. The $\mathrm{Fe}_{3} \mathrm{O}_{4}$ (111) surface unit cell with the in-plane $6 \AA$ periodicity is marked by a black hexagon. Schematics in (c) shows a top view of the $\mathrm{Fe}_{3} \mathrm{O}_{4}(111)$ surface crystallographic structure. In the top view the surface unit cell with a lattice constant of $5.96 \AA$ (black hexagon) and of the oxygen-sublattice surface unit cell with a lattice constant of $2.98 \AA$ (white hexagon) are represented.

Surface characterization of the in situ prepared $\mathrm{Fe}_{3} \mathrm{O}_{4}(111)$ films was performed by means of x-ray absorption spectroscopy (XAS), low-energy electron diffraction (LEED), and scanning tunnelling microscopy (STM) as shown in figure 2 [32]. Panel (a) presents the $\mathrm{Fe} \mathrm{L}_{2,3^{-}}$ edge XAS spectra of the epitaxial $\mathrm{Fe}_{3} \mathrm{O}_{4}(111)$ film as well as of the epitaxial $\mathrm{Fe}(110)$ film. The characteristic multiplet structure of the XAS spectrum at the $\mathrm{Fe} \mathrm{L}_{2,3}$ of $\mathrm{Fe}_{3} \mathrm{O}_{4}$ agrees with earlier experiments [33-35] confirming the high quality of the films. In the insets in figure 2(a) the corresponding LEED patterns of the $\mathrm{Fe}_{3} \mathrm{O}_{4}(111)$ and $\mathrm{Fe}(110)$ surfaces are shown. The hexagonal $(2 \times 2)$ LEED pattern indicates the formation of a $\mathrm{Fe}_{3} \mathrm{O}_{4}(111)$ surface [36, 35, 26, 29, 30]. Figure 2(b) shows an atomically resolved STM image of the regular defect-free $\mathrm{Fe}_{3} \mathrm{O}_{4}$ (111) surface. A hexagonal lattice with a $6 \AA$ in-plane periodicity and a corrugation amplitude of about $0.5 \AA$ was observed. The in-plane value of the $6 \AA$ measured by STM fits well with the $\mathrm{Fe}_{3} \mathrm{O}_{4}(111)$ in-plane lattice constant of $5.92 \AA$ (see figure 2(c)). The observed structure of the $\mathrm{Fe}_{3} \mathrm{O}_{4}(111)$ surface is in good agreement with LEED measurements as well as with previous studies [37]. These data confirm the high bulk and surface quality of the investigated $\mathrm{Fe}_{3} \mathrm{O}_{4}(111)$ films.

The spin-resolved photoemission spectra together with the total emission intensity and the spin polarization as a function of binding energy for the $\mathrm{Fe}_{3} \mathrm{O}_{4}(111)$ and $\mathrm{Fe}(110)$ films prepared on W(110) are presented in figure 3. The spin-resolved spectra of the valence band of $\mathrm{Fe}(110)$ (bottom curves in the left-hand panel of figure 3) show the emission from the $\Sigma^{1} \downarrow \otimes \Sigma^{3} \downarrow$ states near $0.25 \mathrm{eV}$ and from the $\Sigma^{1} \uparrow \otimes \Sigma^{4} \uparrow$ states near $0.7 \mathrm{eV}$. The spectra are in agreement with previous measurements $[38,39]$. A spin polarization value of about $-(80 \pm 5) \%$ at $E_{\mathrm{F}}$ at room temperature (RT) can be observed on the $\mathrm{Fe}(110)$ surface.

The spin-resolved spectra of the $\mathrm{Fe}_{3} \mathrm{O}_{4}(111)$ film on the $\mathrm{Fe}(110) / \mathrm{W}(110)$ system measured near $E_{\mathrm{F}}$ at RT clearly show a dominant emission from the spin-down $\mathrm{Fe} 3 \mathrm{~d}$ 


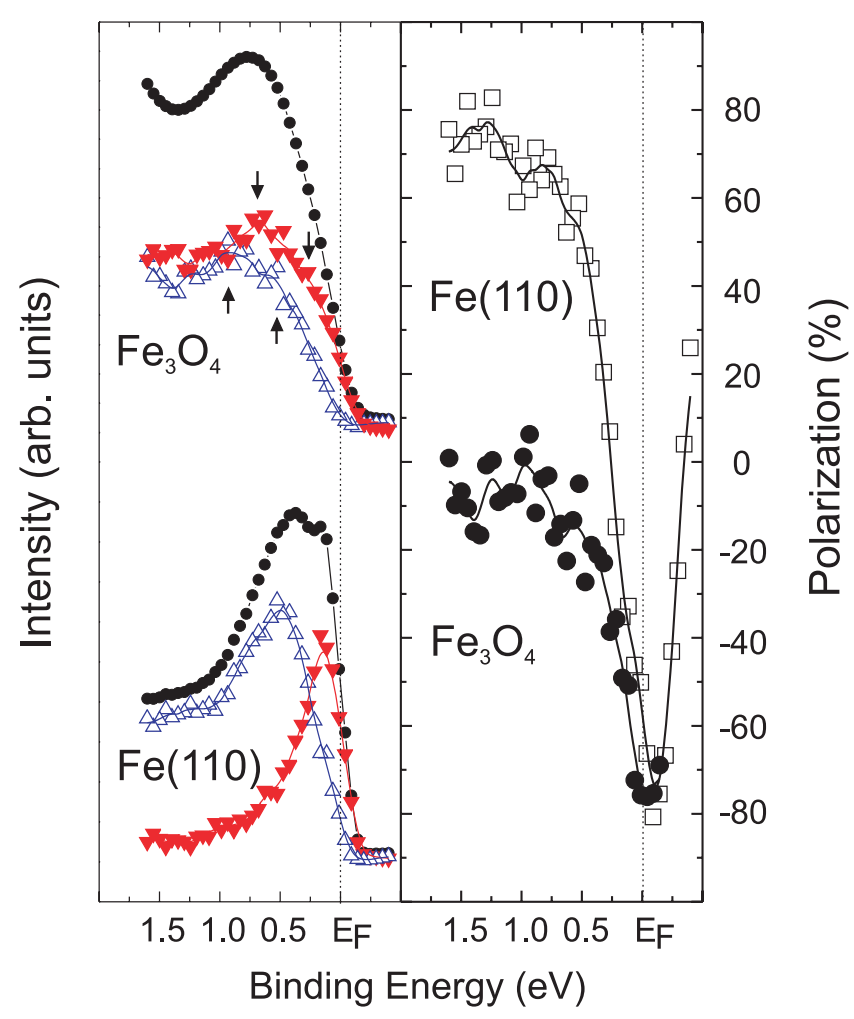




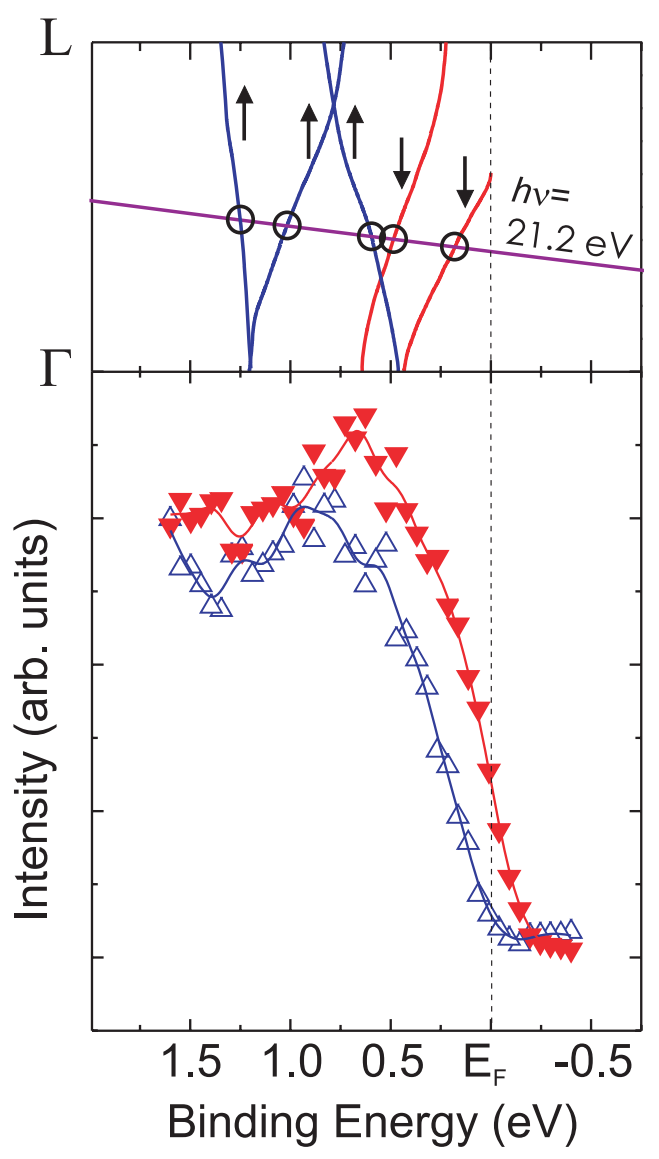

Figure 4. Lower panel shows the spin-resolved photoemission spectra of $\mathrm{Fe}_{3} \mathrm{O}_{4}(111)$ for $h v=$ $21.2 \mathrm{eV}$ in normal emission from figure 3. In the upper panel the spin-split bands of $\mathrm{Fe}_{3} \mathrm{O}_{4}$ along the $\Gamma-\mathrm{L}$ direction from [2] are presented. The free-electron-like final state has been shifted down by $21.2 \mathrm{eV}$ to yield crossing points indicating the initial states in photoemission.

In the photoemission spectra in figure 3, only features of $\mathrm{Fe}_{3} \mathrm{O}_{4}$ are present with a negligible contribution from the $\mathrm{Fe}(110)$ substrate. This is further corroborated by a comparison with the spin-split electron band structure of $\mathrm{Fe}_{3} \mathrm{O}_{4}$ based on the LSDA to the 'constrained' density-functional method [2]. For the $\mathrm{Fe}_{3} \mathrm{O}_{4}(111)$ layers, a reduced photoemission intensity near $E_{\mathrm{F}}$ is observed compared to the pure $\mathrm{Fe}(110)$ film (see figure 3): for spin-up electrons more than 20 times and for spin-down electrons more than eight times. This can be interpreted as a band gap formation for spin-up electrons near $E_{\mathrm{F}}$ for $\mathrm{Fe}_{3} \mathrm{O}_{4}(111) / \mathrm{Fe}(110)$ which is also consistent with the band structure calculation [2] showing a $0.45 \mathrm{eV}$ gap for the spin-up electrons below $E_{\mathrm{F}}$.

The detailed features in the spin-resolved photoemission spectra of $\mathrm{Fe}_{3} \mathrm{O}_{4}(111)$ indicated by arrows in figure 3 are compared in figure 4 (lower panel) with the band dispersion in $\Gamma-\mathrm{L}$ direction (figure 4, upper panel) as calculated in [2]. For the final state in the photoemission using $h v=21.2 \mathrm{eV}$ we assumed a free-electron-like dispersion with a spin averaged inner potential of $3.5 \mathrm{eV}$ [36]. The downshift of the final-state dispersion by $21.2 \mathrm{eV}$ leads to crossing points with the spin-split conduction bands (figure 4, upper panel), which can be related to features in the spin-resolved photoemission spectra (figure 4, lower panel). The crossing points 
in the spin-down bands near 0.2 and $0.5 \mathrm{eV}$ correspond to the shoulder near $0.25 \mathrm{eV}$ and the broad maximum near $0.6 \mathrm{eV}$, respectively, in the spin-down spectrum. In the spin-up bands the crossing points near $0.6,1.0$, and $1.25 \mathrm{eV}$ are related to, respectively, the shoulder near $0.5 \mathrm{eV}$, the broad maximum near $0.9 \mathrm{eV}$, and the slight shoulder near $1.25 \mathrm{eV}$ in the spin-up spectrum. The credibility of these assignments is further supported by the tight-binding fit to the linear muffin tin orbital (LMTO) minority bands [2]. The band dispersion is not drastically changed upon going from the $\Gamma$ point halfway to the $\mathrm{L}$ point. The two crossing points in the spin-down bands in the upper panel of figure 4 are shifted by 0.1 to $0.2 \mathrm{eV}$ to higher binding energies. The agreement with features in the spin-down spectra is slightly better.

The spin-resolved photoemission spectra of $\mathrm{Fe}_{3} \mathrm{O}_{4}(111)$ for binding energies $1.5 \mathrm{eV} \leqslant$ $E_{\mathrm{b}} \leqslant E_{\mathrm{F}}$ support the band-type description of the electronic structure of $\mathrm{Fe}_{3} \mathrm{O}_{4}$. Within this energy interval the photoemission spectra were previously attributed to the ionic configurationbased transition ${ }^{5} \mathrm{~T}_{2}\left(3 \mathrm{~d}^{6}\right) \rightarrow{ }^{6} \mathrm{~A}_{1}\left(3 \mathrm{~d}^{5}\right)+\mathrm{e}^{-}$of $\mathrm{Fe}^{2+}$, where $\mathrm{e}^{-}$denotes a photoelectron. The maximum obtainable spin polarization at $T=0 \mathrm{~K}$ of $P=-2 / 3$ or $-66.6 \%$ derived in this model [25], however, cannot account for our experimental finding of $P=-(80 \pm 5) \%$ at room temperature. Hence, despite the importance of electron correlation effects in transition metal oxides, a band-type description of the electronic structure of $\mathrm{Fe}_{3} \mathrm{O}_{4}$ seems to be appropriate. Similar band dispersions have been found for $1.0 \mathrm{eV}<E_{\mathrm{b}} \leqslant E_{\mathrm{F}}$ for $\mathrm{CrO}_{2}$ in a LSDA $+U$ approach [43]. The agreement of the spin-resolved data with density functional calculations provides the evidence for the half-metallic ferromagnetic state of $\mathrm{Fe}_{3} \mathrm{O}_{4}$ in the [111] crystallographic direction.

\section{Spin-resolved photoelectron spectroscopy of $\mathrm{Fe}_{3} \mathrm{O}_{4}(100)$}

High quality epitaxial $\mathrm{Fe}_{3} \mathrm{O}_{4}(100)$ films were prepared in situ by $\mathrm{Fe}$ evaporation on $\mathrm{MgO}(100)$ in $\mathrm{O}_{2}$ atmosphere. During the growth, the $\mathrm{O}_{2}$ pressure was maintained at $2 \times 10^{-6}$ mbar and moderate substrate temperatures of about $300{ }^{\circ} \mathrm{C}$ were used to prevent magnesium interdiffusion in the case of $\mathrm{MgO}(100)$ substrates. The surface characterization of the in situ prepared $\mathrm{Fe}_{3} \mathrm{O}_{4}(100)$ films was performed by means of XAS, LEED, and STM as shown in figure 5. The Fe $\mathrm{L}_{2,3}$-edge XAS spectrum showed the same multiplet structure as presented in figure 2, indicating a good agreement with earlier experiments [34, 35] which confirms the good bulk quality of the prepared samples. Atomically flat terraces with step edges of non-defined shape can be clearly seen in figure 5(a), revealing a layer-by-layer growth of $\mathrm{Fe}_{3} \mathrm{O}_{4}(100)$. The inset in figure 5(a) shows a typical LEED pattern of the (100)-oriented surface of an epitaxial $\mathrm{Fe}_{3} \mathrm{O}_{4}$ film grown on an $\mathrm{MgO}(100)$ substrate. A clear $(\sqrt{2} \times \sqrt{2}) \mathrm{R} 45^{\circ}$ reconstruction with respect to the (100)-unreconstructed $\mathrm{Fe}_{3} \mathrm{O}_{4}$ surface unit cell is visible. In the high-resolution STM image of a typical terrace (figure 5(b)) rows consisting of bright protrusions are imaged. The distance between two bright protrusions within the same row was measured to be about $3 \AA$, and the distance between two rows is about $6 \AA$. Alternating pairs of bright protrusions within each row are shifted into the same direction perpendicular to the rows, resulting in a wave-like surface structure. Regularly ordered large dark depressions appear at the wide openings of the waves. The centres of these depressions are separated by $8.4 \AA$ and have a $(\sqrt{2} \times \sqrt{2}) \mathrm{R} 45^{\circ}$ symmetry. Similar square-lattice structure types were also observed in STM measurements by Stanka et al [44].

In order to resolve the structure and composition of the surface layer, we performed DFT calculations for a variety of stoichiometric and nonstoichiometric models of $\mathrm{Fe}_{3} \mathrm{O}_{4}$. Here, the DFT calculations were performed using the full-potential augmented plane waves (FP-APW) method in the WIEN2K implementation [45] within the generalized gradient approximation (GGA) [46] of the exchange-correlation functional. The details of the calculation can be 

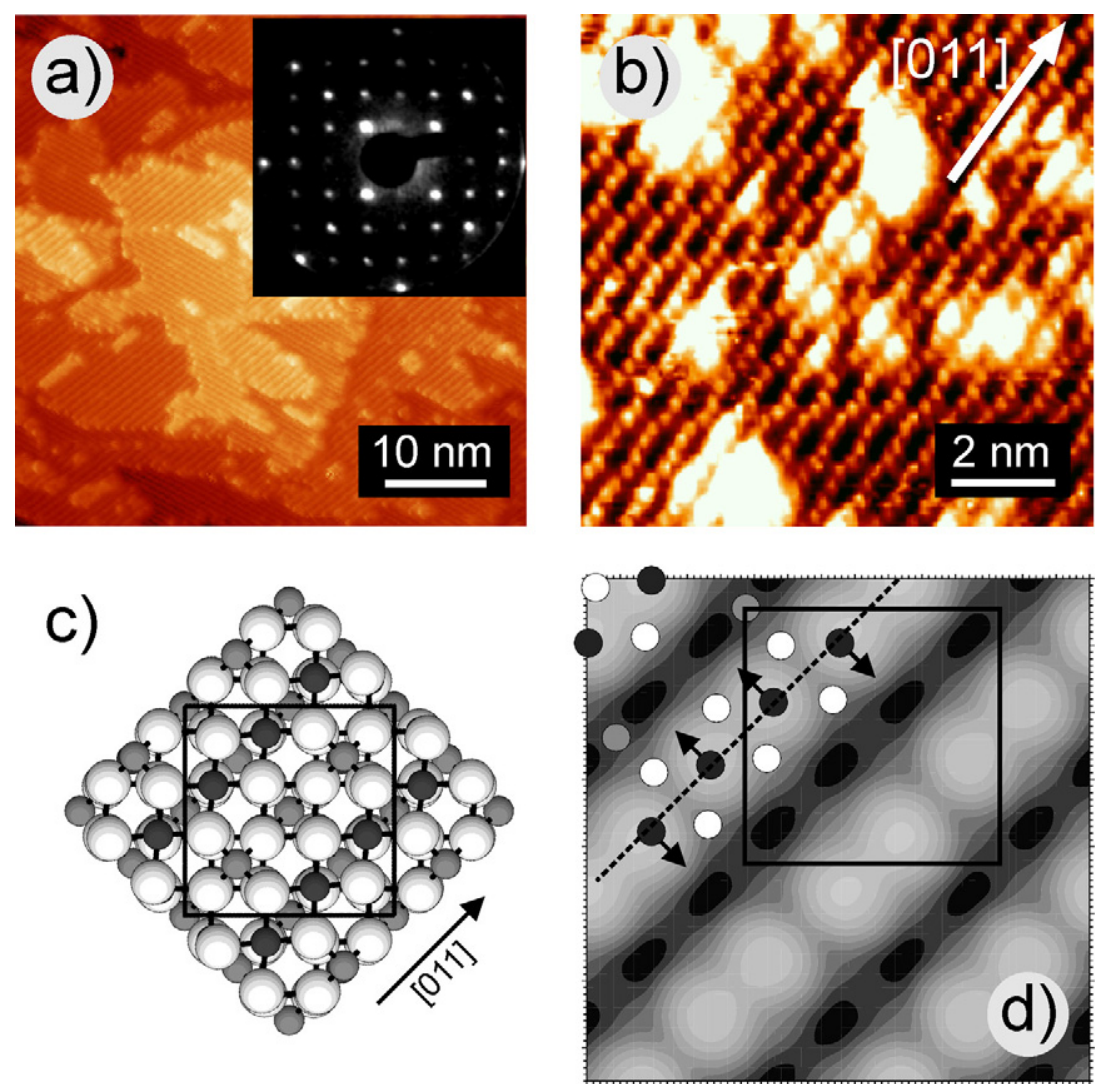

Figure 5. (a) $50 \times 50 \mathrm{~nm}^{2} \mathrm{STM}$ image of a $(\sqrt{2} \times \sqrt{2}) \mathrm{R} 45^{\circ}$ reconstructed $\mathrm{Fe}_{3} \mathrm{O}_{4}(100)$ surface The inset shows a typical $(\sqrt{2} \times \sqrt{2}) \mathrm{R}_{4} 5^{\circ}$ LEED diffraction pattern of a $\mathrm{Fe}_{3} \mathrm{O}_{4}(100)$ surface. (b) High-resolution STM image $\left(11 \times 11 \mathrm{~nm}^{2}\right)$ of the $\mathrm{Fe}_{3} \mathrm{O}_{4}(100)$ surface showing a wave-like atomic structure which gives rise to a $(\sqrt{2} \times \sqrt{2}) \mathrm{R} 45^{\circ}$ periodicity. (c) A top view of the modified B-termination predicted from the DFT-calculations [47]. (d) Simulated STM image of the modified B-termination. The positions of the surface oxygen, octahedral iron and subsurface tetrahedral iron are marked by white, grey and black circles, respectively. The black open square in (c) and (d) denotes the $(\sqrt{2} \times \sqrt{2}) \mathrm{R} 45^{\circ}$-unit cell. The dashed line in (d) is a guide to the eyes to emphasize the displacements of surface $\mathrm{Fe}_{\mathrm{B}}$ atoms perpendicular to the [011]-direction.

found elsewhere [31]. A surface phase diagram of $\mathrm{Fe}_{3} \mathrm{O}_{4}(100)$ as a function of the oxygen pressure was compiled in the framework of $a b$ initio atomistic thermodynamics, indicating that a modified bulk termination with a B layer showing a wave-like structure (figure 5(c)) has the lowest energy over a broad range of oxygen pressures [47]. We performed STM simulations of the modified B termination predicted by DFT calculations in order to interpret the atomically resolved STM images. The simulated STM image in figure 5(d) is obtained in the constant current mode using the Tersoff-Hamann approximation [48]. The charge density from the DFT calculation was integrated from $E_{\mathrm{F}}$ to $E_{\mathrm{F}}+1.1 \mathrm{eV}$, which corresponds to the voltage used in the STM measurements presented above. In figure 5(d) bright protrusions mark the positions of the surface $\mathrm{Fe}_{\mathrm{B}}$. As indicated by the arrows, alternating pairs of such protrusions are shifted perpendicular to the [011] direction giving rise to a wave-like pattern. Although the wave-like structure is clearly visible in the DFT-derived structure in figure 5(c), it is much less pronounced than in the experimentally obtained STM image (figure 5(b)). This may be attributed to a softness of the surface with respect to vibrational modes. 


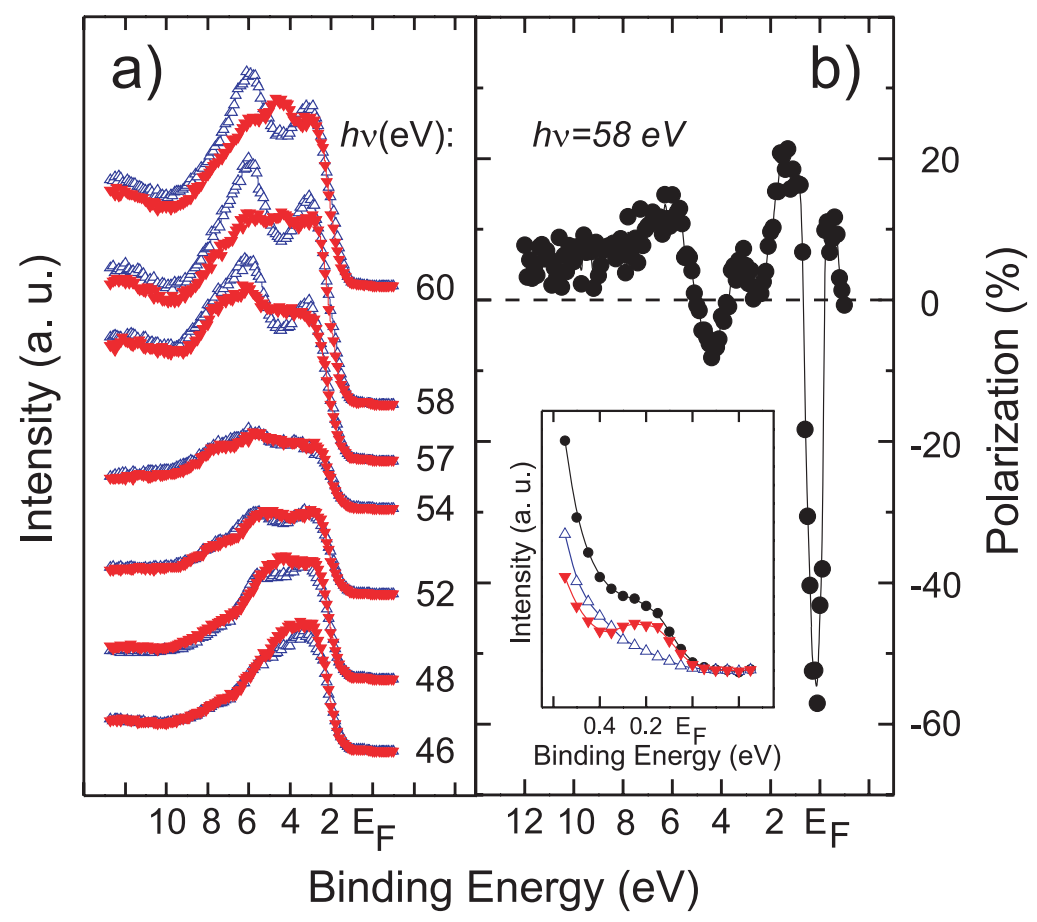

Figure 6. (a) Spin-resolved valence band photoemission spectra (spin-up: open triangle up; spindown: solid triangle down) of a $\mathrm{Fe}_{3} \mathrm{O}_{4}(100)$ thin film on $\mathrm{MgO}(100)$ as a function of binding energy recorded at RT in normal emission at 46-60 eV photon energy. (b) Spin polarization recorded with $h v=58 \mathrm{eV}$ as a function of binding energy of the $\mathrm{Fe}_{3} \mathrm{O}_{4}(100) / \mathrm{MgO}(100)$ system. Inset in (b) shows the spin-resolved photoemission spectra of $\mathrm{Fe}_{3} \mathrm{O}_{4}(100)$ in the region near $E_{\mathrm{F}}(h v=58 \mathrm{eV})$.

Figure 6(a) shows representative spin-polarized PES spectra of the $\mathrm{Fe}_{3} \mathrm{O}_{4} / \mathrm{MgO}(100)$ system as a function of binding energy. The position of the Fermi energy corresponds to that of a polycrystalline Au foil in electrical contact with the sample. The spectra were normalized to the current recorded from the last Au-coated mirror of the beamline. The photon energy was tuned over the range of 46-60 eV, including the Fe 3p-3d excitation threshold at $58 \mathrm{eV}$, where a strong resonance behaviour was observed. In the region between 0 and $9 \mathrm{eV}$ of binding energy considerable changes in intensity as well as in spectral shape of the features derived from the $\mathrm{O} 2 \mathrm{p}$ and $\mathrm{Fe} 3 \mathrm{~d}$ states can be observed as a function of the photon energy. Only weak dispersions (if any) of the photoemission features were observed along the $\Gamma-X$ direction of the $\mathrm{Fe}_{3} \mathrm{O}_{4}$ Brillouin zone. In comparison to the present study, well pronounced band dispersions were reported on the $\mathrm{Fe}_{3} \mathrm{O}_{4}(111)$ [49] and $\mathrm{Fe}_{3} \mathrm{O}_{4}(100)$ [50] surfaces which can be due to the better angle resolution of $\pm 1^{\circ}$ compared to the acceptance angle of $6^{\circ}$ used in the present study. In the valence band of $\mathrm{Fe}_{3} \mathrm{O}_{4}(100)$ several photoemission features can be clearly observed: a peak at $0.5 \mathrm{eV}$, a small shoulder at $2.2 \mathrm{eV}$, peaks at $3 \mathrm{eV}$ and $5.9 \mathrm{eV}$, and a shoulder at $7.6 \mathrm{eV}$. The positions as well as the general trend of the resonant enhancement of these features is very similar to those already reported for the $\mathrm{Fe}_{3} \mathrm{O}_{4}(111)$ thin films [49].

Figure 6(b) shows the spin polarization recorded at RT in normal emission with $h v=$ $58 \mathrm{eV}$ as a function of the binding energy relative to the Fermi level of the $\mathrm{Fe}_{3} \mathrm{O}_{4}$ (100) film. The spin-resolved PES spectra in the region near $E_{\mathrm{F}}$ obtained with $h v=58 \mathrm{eV}$ together with the total photoemission intensity is presented in the inset in figure $6(\mathrm{~b})$. The value of $-(55 \pm 10) \%$ 


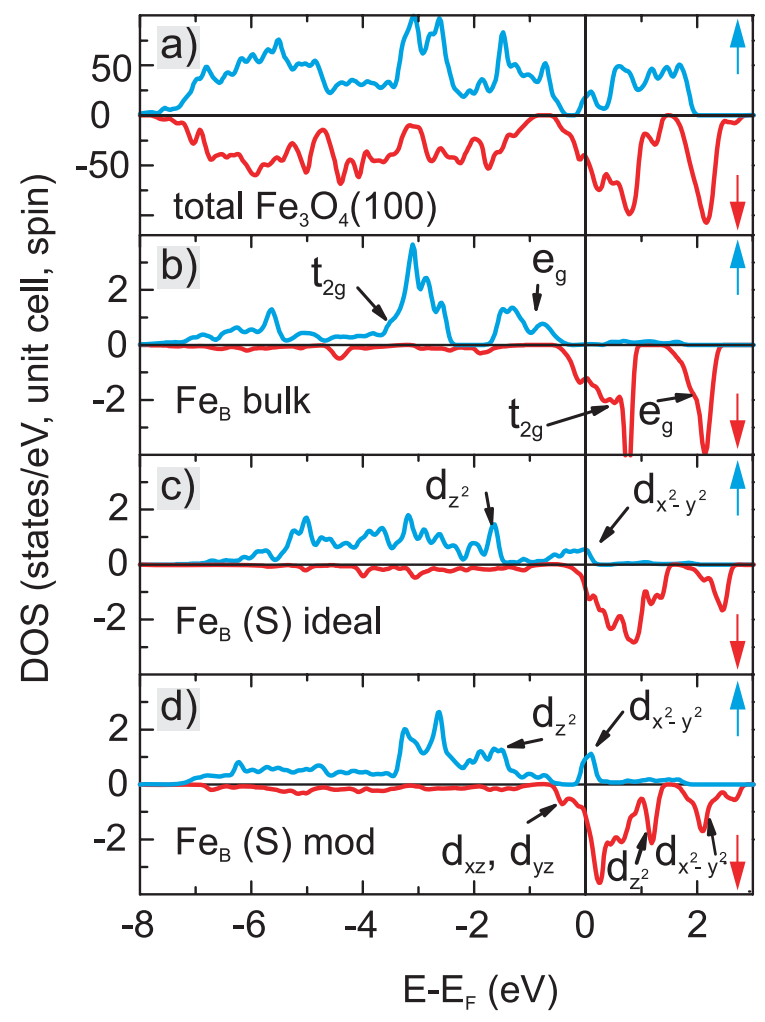

Figure 7. Spin-resolved density of states of the $\mathrm{Fe}_{3} \mathrm{O}_{4}(100)$ surface terminated by a modified Blayer [31]: (a) total DOS; (b) projected DOS of $\mathrm{Fe}_{\mathrm{B}}$ in the bulk; (c) and (d) projected DOS of the surface $\mathrm{Fe}_{\mathrm{B}}$ for the B-termination with ideal (bulk) (c) and relaxed (modified) positions of the atoms, respectively.

at $E_{\mathrm{F}}$ measured on the $\mathrm{Fe}_{3} \mathrm{O}_{4}(100)$ surface at RT is significantly lower than the one for the (111)-surface $(-(80 \pm 5) \%)$ [29] as well as $-100 \%$ predicted by LSDA calculations [2]. However, these band structure calculations were performed for the bulk material, not taking into account surface structure effects, which can prevail in the ultra-violet photoemission spectroscopy measurements.

We have now calculated the surface electronic structure of $\mathrm{Fe}_{3} \mathrm{O}_{4}(100)$ terminated by a wave-like B-layer and the spin-resolved total density of states (DOS) is shown in figure 7(a). The most striking feature of the spin-polarized bands are the states that appear in the band gap of the majority subband leading to a reduction of the spin polarization to about $-40 \%$ at $E_{\mathrm{F}}$ and a loss of half-metallicity at the $\mathrm{Fe}_{3} \mathrm{O}_{4}(100)$ surface. The calculated spin polarization value is somewhat lower than $-(55 \pm 10) \%$ obtained from the photoemission measurements. A possible reason for the difference between the calculated and the experimentally measured spin polarization values may be the finite size of the slab used to model the $\mathrm{Fe}_{3} \mathrm{O}_{4}(100)$. As the states at the Fermi level are relevant for the transport properties of the material, their character was analysed in detail.

In order to gain an understanding of how the electronic structure changes from bulk to the surface as well as to resolve the influence of the surface reconstruction, we have plotted the projected DOS of $\mathrm{Fe}_{\mathrm{B}}$ in the bulk (figure 7(b)) as well as on the surface in the ideal (figure 7(c)) and modified (figure 7(d)) B-termination. In the bulk, the crystal field splitting of the $\mathrm{e}_{\mathrm{g}}$ and 
$\mathrm{t}_{2 \mathrm{~g}}$-states of the octahedrally coordinated iron amounts to $2.0 \mathrm{eV}$. In the majority spin channel both the $\mathrm{e}_{\mathrm{g}}$ and $\mathrm{t}_{2 \mathrm{~g}}$ are occupied while in the minority spin channel the $\mathrm{e}_{\mathrm{g}}$ states are unoccupied and lie $2.0 \mathrm{eV}$ above the Fermi level, whereas $E_{\mathrm{F}}$ crosses the $\mathrm{t}_{2 \mathrm{~g}}$-band. On the surface, due to the broken symmetry in the $z$-direction, the degeneracy within the $e_{\mathrm{g}}$ doublet and $\mathrm{t}_{2 \mathrm{~g}}$ triplet is lifted. In the majority spin channel $\mathrm{d}_{x^{2}-y^{2}}\left(\mathrm{~d}_{z^{2}}\right)$-states are shifted to higher (lower) energies and the former cross the Fermi level even in the ideal (unrelaxed) B-termination (see figure 7(c)).

The DFT results indicate that the origin of the wave-like $(\sqrt{2} \times \sqrt{2}) \mathrm{R} 45^{\circ}$ pattern observed in STM is a Jahn-Teller distortion. The main feature associated with the distortion of the surface atoms is the opening of a band gap in the majority spin channel between -0.4 and

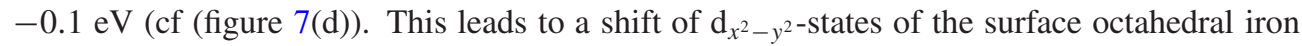
through the Fermi level and a partial deoccupation. The depletion of states in the majority spin channel is accompanied by occupation of states in the minority spin channel. This effect, referred to as spin flip results in a substantial reduction of the magnetic moments of the surface $\mathrm{Fe}_{\mathrm{B}}$ from $3.60 \mu_{\mathrm{B}}$ for the B-termination with bulk positions of the atoms to $3.00 \mu_{\mathrm{B}}$ for the modified B-termination.

As a result of the spin flip effect in the minority spin channel, $\mathrm{d}_{x z}$ - and $\mathrm{d}_{y z}$-states are partially occupied and shifted to lower energies, while $\mathrm{d}_{x y}$-states of the surface iron remain unoccupied at $0.3 \mathrm{eV}$ above $E_{\mathrm{F}}$. The $\mathrm{d}_{z^{2}}$-states are pulled to lower energies at $1.1 \mathrm{eV}$ above the Fermi level almost touching the $t_{2 \mathrm{~g}}$ band and the splitting between $\mathrm{d}_{z^{2}}$ and $\mathrm{d}_{x^{2}-y^{2} \text {-states }}$ amounts to $1.0 \mathrm{eV}$. We note that the positions of the d-orbitals differ from the qualitative picture proposed by Shvets et al [51] which suggests a small and large splitting of the orbitals of $\mathrm{Fe}^{2+}$ and $\mathrm{Fe}^{3+}$, respectively. The authors of [51] conclude that only $\mathrm{Fe}^{2+}$ may be subject to a JahnTeller-distortion. The model of Shvets et al [51] considers the extreme case where the sixth electron is completely localized at $\mathrm{Fe}^{2+}$, while the DFT-GGA calculation renders an average occupation of 5.38 d electrons within the muffin tin for both $\mathrm{Fe}_{\mathrm{B}}$ ions in the surface. Recently, Wright et al [52] obtained evidence from x-ray and neutron powder diffraction that the charge disproportionation between octahedral B iron below the Verwey temperature is 2.4 and 2.6 which is only $20 \%$ of the ideal value for $\mathrm{Fe}^{2+}$ and $\mathrm{Fe}^{3+}$. This suggests that assigning integer charges to the iron ions may not be appropriate. Still, the influence of strong electron correlation effects on the surface electronic structure remains to be investigated.

\section{Conclusion}

In conclusion, the measured spin polarization value of $-(80 \pm 5) \%$ at $E_{\mathrm{F}}$ at RT, rules out the ionic configuration based approach, having set the upper limit of $P=-66.6 \%$ at $T=0 \mathrm{~K}$. The agreement of the photoemission spectroscopy in the [111]-direction with density functional calculations, predicting an overall energy gap in the spin-up electron bands in high symmetry directions, provides evidence for the half-metallic ferromagnetic state of $\mathrm{Fe}_{3} \mathrm{O}_{4}$ in the [111] direction. For the $\mathrm{Fe}_{3} \mathrm{O}_{4}(100)$ surface, both the spin-resolved photoemission experiments and the DFT density of states give evidence for a half-metal to metal transition: the measured negative spin polarization of about $-(55 \pm 10) \%$ at the Fermi level and the theoretical value of $-40 \%$ are significantly lower than the $-100 \%$ predicted by LSDA calculations for the bulk magnetite crystal as well as the $-(80 \pm 5) \%$ obtained for the $\mathrm{Fe}_{3} \mathrm{O}_{4}(111)$ films. The electronic states in the majority spin-channel responsible for the reduction of the spin polarization are dominated by $\mathrm{d}_{x^{2}-y^{2}}$-states of surface $\mathrm{Fe}_{\mathrm{B}}$.

\section{References}

[1] Yanase A and Siratori K 1984 J. Phys. Soc. Japan 53312

[2] Zhang Z and Satpathy S 1991 Phys. Rev. B 4413319 
[3] Verwey E J W 1939 Nature 144327

[4] Verwey E J W and Haaymann P W 1941 Physica 8979

[5] Verwey E J W, Haayman P W and Romeijan F C 1947 J. Chem. Phys. 15181

[6] Hamilton W C 1958 Phys. Rev. 1101050

[7] Samuelsen E J, Bleeker E J, Dobrzynski L and Riste T 1968 J. Appl. Phys. 391114

[8] Yoshida J and Iida S 1979 J. Phys. Soc. Japan 471627

[9] Miyamoto Y and Shindo M 1993 J. Phys. Soc. Japan 621423

[10] Medrano C, Schlrnker M, Baruchel J, Espeso J and Miyamoto Y 1999 Phys. Rev. B 591185

[11] Cullen J R and Callen E R 1971 Phys. Rev. Lett. 26236

[12] Cullen J R and Callen E R 1973 Phys. Rev. B 7397

[13] Zuo J M, Spence J C H and Petuskey W 1990 Phys. Rev. B 428451

[14] Mott N F 1980 Phil. Mag. B 42327

[15] Yamada Y 1980 Phil. Mag. B 42377

[16] Ihle D and Lorenz B 1986 J. Phys. C: Solid State Phys. 195239

[17] Seo H, Ogata M and Fukuyama H 2002 Phys. Rev. B 65085107

[18] Tsuda N, Nasu K, Yanase A and Siratori K 1991 Electronic Conduction in Oxides (Berlin: Springer) p 207

[19] Anisimov V I, Elfimov I S, Hamada N and Terakura K 1996 Phys. Rev. B 544387

[20] Jeng H-T and Guo G Y 2002 Phys. Rev. B 65094429

[21] Antonov V N, Harmon B N, Antropov V P, Perlov A Ya and Yaresko A N 2001 Phys. Rev. B 64134410

[22] Antonov V N, Harmon B N and Yaresko A N 2003 Phys. Rev. B 67024417

[23] Alvarado S F, Eib W, Meier F, Pierce D T, Sattler K, Siegmann H C and Remeika J P 1975 Phys. Rev. Lett. 34319

[24] Alvarado S F, Erbudak M and Munz P 1976 Phys. Rev. B 142740

[25] Alvarado S F and Bagus P S 1978 Phys. Lett. A 67397

[26] Kim H-J, Park J-H and Vescovo E 2000 Phys. Rev. B 6115288

[27] Morton S A, Waddill G D, Kim S, Schuller I K, Chambers S A and Tobin J G 2002 Surf. Sci. 513 L451

[28] Huang D J, Chang C F, Chen J, Tjeng L H, Rata A D, Wu W P, Chung S C, Lin H J, Hibma T and Chen C T 2002 J. Magn. Magn. Mater. 239261

[29] Dedkov Yu S, Rüdiger U and Güntherodt G 2002 Phys. Rev. B 65064417

[30] Fonin M, Dedkov Yu S, Mayer J, Rüdiger U and Güntherodt G 2003 Phys. Rev. B 68045414

[31] Fonin M, Pentcheva R, Dedkov Yu S, Sperlich M, Vyalikh D V, Scheffler M, Rüdiger U and Güntherodt G 2005 Phys. Rev. B 72104436

[32] Dedkov Yu S, Fonin M, Vyalikh D V, Hauch J O, Molodtsov S L, Rüdiger U and Güntherodt G 2004 Phys. Rev. B 70073405

[33] de Groot F M F, Grioni M, Fuggle J C, Ghijsen J, Sawatzky G A and Petersen H 1989 Phys. Rev. B 405715

[34] de Groot F M F, Fuggle J C, Thole B T and Sawatzky G A 1990 Phys. Rev. B 425459

[35] Kim H-J, Park J-H and Vescovo E 2000 Phys. Rev. B 6115284

[36] Barbieri A, Weiss W, van Hove M A and Samorjai G A 1994 Surf. Sci. 302259

[37] Ritter M and Weiss W 1999 Surf. Sci. 43281

[38] Kurzawa R, Kämper K-P, Schmitt W and Güntherodt G 1986 Solid State Commun. 60777

[39] Kim H-J and Vescovo E 1998 Phys. Rev. B 5814047

[40] Gong G Q, Gupta A, Xiao G, Qian W and Dravid V P 1997 Phys. Rev. B 565096

[41] Alvarado S F 1979 Z. Phys. B 3351

[42] Mauri D, Scholl D, Siegmann H C and Kay E 1988 Phys. Rev. Lett. 61758

[43] Korotin M A, Anisimov V I, Khomskii D I and Sawatzky G A 1998 Phys. Rev. Lett. 804305

[44] Stanka B, Hebenstreit W, Diebold U and Chambers S A 2000 Surf. Sci. 44849

[45] Blaha P, Schwarz K, Madsen G K H, Kvasnicka D and Luitz J 2001 WIEN2k, An Augmented Plane Wave + Local Orbitals Program for Calculating Crystal Properties (Karlheinz Schwarz, Techn. Universität Wien, Austria) ISBN 3-9501031-1-2 http://www.wien2k.at

[46] Perdew J P, Burke K and Ernzerhof M 1996 Phys. Rev. Lett. 773865

[47] Pentcheva R, Wendler W, Meyerheim H L, Moritz W, Jedrecy N and und Scheffler M 2005 Phys. Rev. Lett. 94126101

[48] Tersoff J and Hamann D R 1985 Phys. Rev. B 31805

[49] Cai Y Q, Ritter M, Weiss W and Bradshaw A M 1998 Phys. Rev. B 585043

[50] Dedkov Yu S, Fonin M, Rüdiger U and Güntherodt G, unpublished

[51] Shvets I V, Mariotto G, Jordan K, Berdunov N, Kantor R and Murphy S 2004 Phys. Rev. B 70155406

[52] Wright J P, Attfield J P and Radaelli P G 1996 Phys. Rev. Lett. 773865 